\title{
Steuerung braucht Lotsen
}

\section{Strategische Sozialplanung für Freie Träger}

ANDREAS BECK

Andreas Beck ist Leiter der Machbarkeitsstudie »Implementierung eines strategischen Zentrums für Sozialplanung " der LIGA der Freien Wohlfahrtspflege in Thüringen e. V. abeck@paritaet-th.de

\author{
Die Sozialwirtschaft ist ein weitgehend \\ konjunkturunabhängiger Wirtschaftsbereich, der \\ selbst in den letzten Krisenjahren noch gewachsen \\ ist. Aufgrund der demografischen Entwicklung \\ wird erwartet, dass die Nachfrage nach sozialen \\ Dienstleistungen weiter steigt. Zwei Studien aus \\ Thüringen zeigen, was dies für die Gestaltung sozialer \\ Verbände und Unternehmen bedeuten kann.
}

Am 16. Mai 2012 fand in Erfurt der 1. Thüringer Sozialwirtschaftskongress statt. Die Thüringer Ministerien für Soziales, Familie und Gesundheit sowie Wirtschaft, Arbeit und Technologie, die LIGA der Freien Wohlfahrtspflege in Thüringen e. V. und die FriedrichEbert-Stiftung Thüringen hatten die Tagung organisiert - und erzeugten damit großes Interesse.

Dieser Artikel ist keine »Nacherzählung « der Veranstaltung, sondern geht einer gezielten Nachfrage aus dem Fachpublikum nach. Dazu ist hilfreich zu wissen, dass an diesem Tag zwei aktuelle Publikationen zur Thüringer Sozialwirtschaft eine wichtige Rolle spielten:

- Anlässlich des 1. Thüringer Sozialwirtschaftskongresses wurde der "Sozialwirtschaftsbericht Thüringen« (1) veröffentlicht und vorgestellt. Die zentralen Ergebnisse daraus waren Themen der folgenden Programmpunkte.

- Ende Februar 2012 wurde die »Machbarkeitsstudie zur Implementierung eines Strategischen Zentrums für Sozialplanung« (2) der LIGA Thüringen abgeschlossen und auch deren Ergebnisse und Handlungsempfehlungen waren Gegenstand der Befassung.
Dabei wurde die Frage gestellt: Welchen wechselseitigen Bezug gibt es zwischen den beiden Studien? Nach Überzeugung des Verfassers besteht ein enger Zusammenhang zwischen beiden Untersuchungen, so unterschiedlich sie vom Aufbau sind. Um dies zu verdeutlichen, werden beide Publikationen kurz porträtiert.

\section{Der "Sozialwirtschaftsbericht Thüringen«}

wurde im Auftrag der Thüringer Ministerien für Soziales, Familie und Gesundheit sowie Wirtschaft, Arbeit und Technologie beim Institut für Arbeits-, Industrie- und Wirtschaftssoziologie der Friedrich-Schiller-Universität Jena in Auftrag gegeben. Neben der gezielten Auswertung bestehender Datenquellen, beispielsweise der Statistikbehörden des Bundes und des Freistaats Thüringen, wurden durch telefonische Befragungen Leitungskräfte der freigemeinnützigen, der privaten und der öffentlichen Sozialwirtschaft zu Themen der Beschäftigung und Entlohnung, der Fachkräftesituation, betriebswirtschaftlicher Eckdaten und Finanzierungsbedingungen befragt. Auch die Bedeutung ehrenamtlichen Engagements in der Sozialwirtschaft spielte eine Rolle. Mithilfe der 


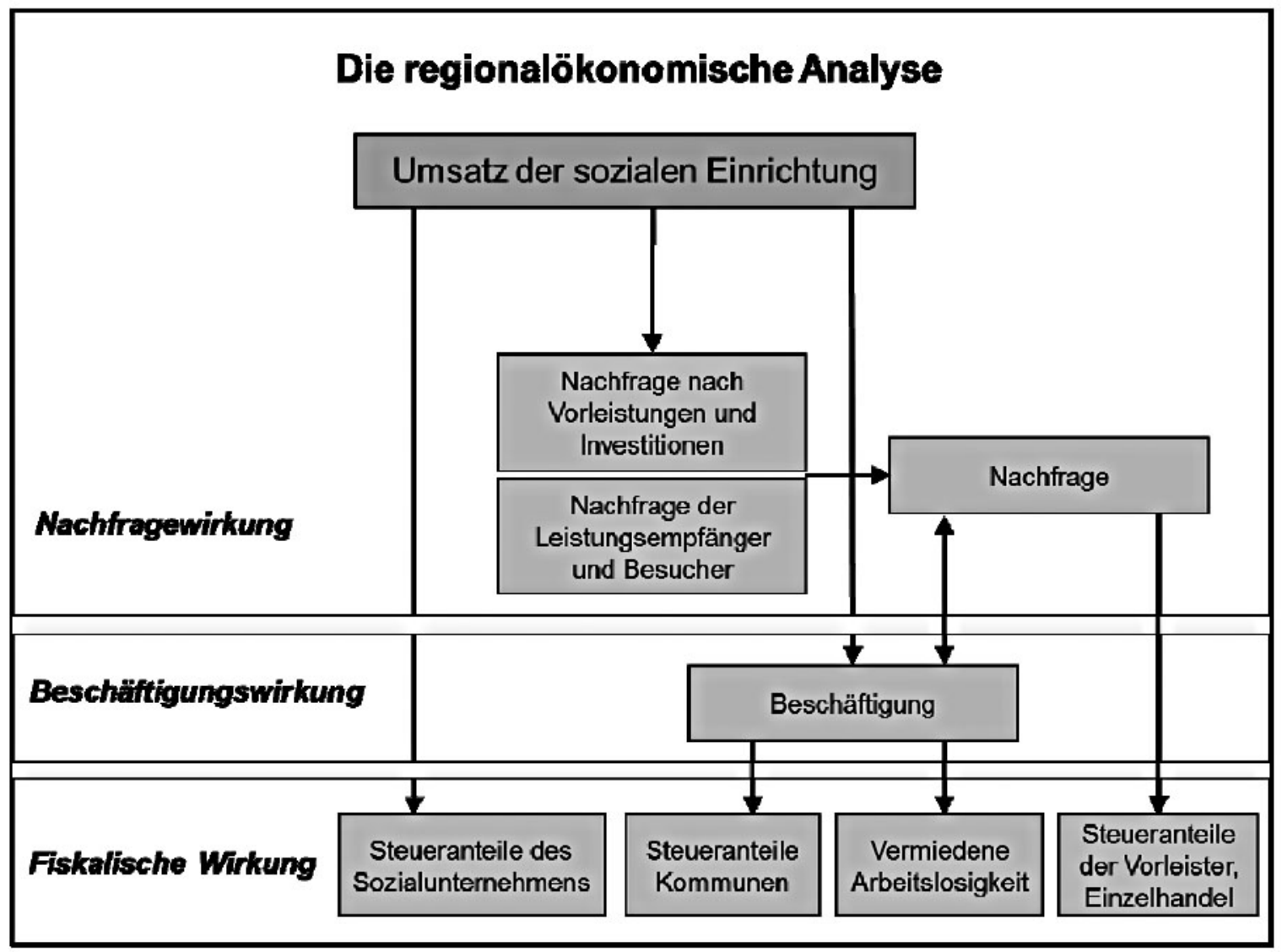

Soziale Dienste kosten nicht nur Geld, sondern bringen auch ökonomischen Gewinn für die Region, wie beispielhaft der "Sozialwirtschaftsbericht Thüringen« darlegt.

www.thueringen.de/de/tmsfg/aktuell/59952/content.html

Evangelischen Fachhochschule Nürnberg (Prof. Dr. Klaus Ulrich Schellberg) wurde ferner ein regionalökonomisches Modell zur Verflechtung der Sozialwirtschaft mit anderen Gesellschaftsteilen errechnet.

Im Ergebnis belegt der Sozialwirtschaftsbericht zum einen die große wirtschaftliche Bedeutung der Sozialwirtschaft in Thüringen: Im Jahre 2010 waren 6,5 Prozent der sozialversicherungspflichtigen Beschäftigten Thüringens in der Sozialwirtschaft tätig; es besteht eine stabile Beschäftigungssteigerung. Im Jahre 2010 wurde eine Bruttowertschöpfung von rund zwei Milliarden Euro erzielt, was etwa 4,7 Prozent der gesamten Thüringer Bruttowertschöpfung ausmacht. Zum anderen weist die Studie aber auch explizit auf dringenden Handlungsbedarf für eine positive Entwicklung der Sozialwirtschaft hin: Fachkräftebedarf, Entlohnungsunterschiede zu den westdeutschen Bundesländern usw.

\section{Die Machbarkeitsstudie zur Strategischen Sozialplanung}

wurde wenige Wochen vor dem Sozialwirtschaftsbericht von der LIGA Thüringen vorgestellt. (3)

Dass sich die Thüringer Spitzenverbände überhaupt mit dem Thema Sozialplanung befassen, lässt sich mit der spezifischen Perspektive darauf verstehen, was »Sozialplanung « im Sinne der Machbarkeitsstudie bedeutete, nämlich: Strategische Sozialplanung als die langfristige, planmäßige und bedarfsgerechte Weiterentwicklung der sozialen Infrastruktur, wobei der Beteiligung von Expertinnen und Experten in eigener Sache und der Freien Träger und deren Spitzenverbände besondere Aufmerksamkeit zukam.

Die Leitfrage der Untersuchung war: Kann ein als »Kompetenzzentrum Strategische Sozialplanung « bezeichneter Ressourcenpool aller LIGA-Verbände hilfreich sein, um Freie Träger in der partizipativen Entwicklung der sozialen Infrastruktur vor Ort zu unterstützen? Und kann gleiches für Planungsprozesse auf überörtlicher und landesweiter Ebene funktionieren?

Ein solches Kompetenzzentrum der LIGA könnte u. a. Planungswissen und Beispiele guter Praxis zusammentragen und weitervermitteln, Qualifizierungsmodule zu Methoden (strategischer) Sozialplanung, zu wirksamen Beteiligungsformen für die Adressaten sozialer Arbeit und für die freigemeinnützigen Träger anbieten, komplexe Planungen durch Prozessmanagement und - wo gewünscht - durch Moderation unterstützen, und die Vernetzung innerhalb der LIGA-Strukturen sowie mit relevanten Akteuren der Politik und Administration lebendig erhalten.

In einer bundesweit neuartigen $\mathrm{Zu}$ sammenarbeit der LIGA-Verbände ging ein interdisziplinäres, dreiköpfiges Team diesen Überlegungen nach. Naturgemäß war dies ein suchender 
Prozess, in dem sowohl theoretische Recherchearbeit wie auch mit hohem Anteil Erprobung in der Praxis stattfand.

Letzteres meint einen fortwährenden Dialog mit Planungsexperten der Thüringer Sozialplanung (z. B. in den Kommunen und mit der Stabsstelle Strategische Sozialplanung im Ministerien für Soziales, Familie und Gesundheit) und einem zwölfköpfigen Beirat, der
Über Jahre hinweg war die Sozialwirtschaft insgesamt ein "schlafender Riese", der nun langsam munter wird und selbstbewusster auftritt. Ihre bunte Vielfalt legt aber noch ein weiteres Bild nahe: Die Wohlfahrtsverbände und ihre Untergliederungen erscheinen bei aller unterschiedlicher Strukturierung als ein ganzer »Flottenverband großer Tanker, bunt beladener Containerschiffe und

\section{"Die Wohlfahrtsverbände gleichen nicht großen Tankern, sondern eher Flottenverbänden«}

sich aus Führungspersönlichkeiten der LIGA, der Ministerien für Soziales, Familie und Gesundheit sowie Wirtschaft, Arbeit und Technologie, der Wirtschaft, der Forschung, des Thüringer Landesrechnungshofs und der kommunalen Sozialverwaltung zusammensetzte.

Die Praxisnähe wurde durch aktive Mitarbeit in laufenden Prozessen erzielt, die die Thüringer Sozialwirtschaft während der Studiendauer prägten: Dabei ging es beispielsweise um die Weiterentwicklung der Eingliederungshilfe für Menschen mit Behinderung, um soziale Infrastruktur in kleinen Gemeinden, um Sozialberichterstattung oder um den Entwurf des Thüringer Landesentwicklungsprogramms 2025.

\section{Sozialwirtschaft als schlafender Riese}

Und wo liegt nun der Bezug zwischen dem »Sozialwirtschaftsbericht Thüringen " und der "Machbarkeitsstudie Strategische Sozialplanung «? Und was hat das mit dem Thema Steuerung in der Sozialen Arbeit zu tun?

Der Sozialwirtschaftsbericht belegt, dass - im Sinne der Subsidiarität wenig überraschend - ein großer Anteil der sozialen und sozialwirtschaftlichen Infrastruktur in der Hand Freier Träger liegt. Angesichts der mindestens 58.000 Beschäftigten, vieler tausender Ehrenamtlicher, der regionalwirtschaftlichen Bedeutung und der beeindruckenden finanziellen Volumina, die der dritte Sektor bewegt, wird die gesamtgesellschaftliche Verantwortung der Freien Wohlfahrtspflege mehr als deutlich. wendiger Boote", die in einem geografisch abgesteckten und dynamischen »Seegebiet « des Freistaats Thüringen kreuzen.

Wohlgemerkt ist diese Flottenbewegung kein Selbstzweck, sondern unverzichtbarer Bestandteil einer funktionierenden Gesamtgesellschaft. Das Bild macht klar, dass »Steuern auf Sicht" unverantwortbar wäre. Wilde Regatten einzelner Träger oder gar Piraterie erscheinen vielleicht auf subjektiver Ebene und bei kurzfristiger Orientierung als rational, gefährden aber genauso wie unbeabsichtigte Kollisionen und Havarien infolge unzureichender Wartung die Sicherheit aller Schiffe und ihrer Besatzungen sowie die Zielerreichung der zivilgesellschaftlichen Auftraggeber.

Sozialplanung als Methode und Instrument der Steuerungsunterstützung kann und soll niemals die Funktion eines Kapitäns oder gar Flottenadmirals einnehmen. Der maritime Vergleich legt aber eine sehr anspruchsvolle Rolle der Sozialplanung nahe: die des Lotsen, der Untiefen und Strömungen kennt, einen geschulten Blick auf das Wetter und die übrigen Schiffsbewegungen hat und so praxistaugliche Hinweise zur Steuerung geben kann.

Der Lotse hilft dem Kapitän, sein Ziel zu erreichen - vielleicht nicht immer auf dem schnellsten Wege, aber so sicher wie möglich. Folgt man dem Ansatz eines gemeinsamen LIGAKompetenzzentrums für Strategische Sozialplanung, ist auch dieser Aspekt in dem Bild gut darzustellen: Ein Lotse ist kein Mitglied der Stammbesatzung, sondern kommt punktgenau an Bord.
Seine Arbeit gewinnt dadurch, dass er viele Schiffstypen kennt und viele Kapitäne berät.

\section{Resümee}

Die Ergebnisse der Machbarkeitsstudie untermauern anhand vieler Beispiele, wie sinnvoll ein weiteres arbeitsteiliges Vorgehen der LIGA zur Strategischen Sozialplanung in Thüringen ist. Der Ansatz eines gemeinsamen Kompetenzzentrums zeigt sich im Rückblick auf achtzehn ereignisreiche Studienmonate als sinnvoll und hilfreich. Tatsächlich haben die Thüringer Wohlfahrtsverbände diesen Gedanken teilweise aufgegriffen und im Mai 2012 eine Stabsstelle für Sozialplanung bei der LIGA-Geschäftsstelle in Erfurt eingerichtet. (4)

\section{Anmerkungen}

(1) $\mathrm{Zu}$ finden im Internet unter: http:// www.thueringen.de/de/tmsfg/aktuell/59952/content.html.

(2) $\mathrm{Zu}$ finden im Internet unter: http:// www.liga-thueringen.de/Meldung. 136.0.html? \&style = \&layout $=\& \mathrm{tx}$ ttnews[pointer]=1\&tx_ttnews[tt news]=454\&tx_ttnews[backPid] $=1$ $35 \& \mathrm{cHash}=47 \mathrm{a} 8 \mathrm{f} 86 \mathrm{de} 1$.

(3) Zu finden im Internet unter http:// www.liga-thueringen.de.

(4) Kontakt: info@liga-thueringen.de, Telefon 0361 511499-20. 